\title{
Brouillage des frontières, rencontres des domaines : quelles conséquences pour l'enseignement de la terminologie et de la traduction spécialisée
}

\section{Geneviève Bordet}

\section{OpenEdition \\ Journals}

\section{Édition électronique}

URL : http://journals.openedition.org/asp/3851

DOI : 10.4000/asp.3851

ISSN : 2108-6354

\section{Éditeur}

Groupe d'étude et de recherche en anglais de spécialité

\section{Édition imprimée}

Date de publication : 1 novembre 2013

Pagination : $95-115$

ISSN : 1246-8185

Référence électronique

Geneviève Bordet, «Brouillage des frontières, rencontres des domaines : quelles conséquences pour l'enseignement de la terminologie et de la traduction spécialisée ", ASp [En ligne], 64 | 2013, mis en ligne le 01 novembre 2014, consulté le 10 décembre 2020. URL : http://journals.openedition.org/asp/ 3851 ; DOI : https://doi.org/10.4000/asp.3851

Ce document a été généré automatiquement le 10 décembre 2020.

Tous droits réservés 


\title{
Brouillage des frontières, rencontres des domaines : quelles conséquences pour l'enseignement de la terminologie et de la traduction spécialisée
}

\author{
Geneviève Bordet
}

1 L'enseignement de la traduction spécialisée s'appuie nécessairement sur une approche terminologique rigoureuse, même si cette approche ne saurait résumer l'ensemble des questions posées par la traduction, ou le processus de transfert interculturel d'un discours qu'elle recouvre. Or c'est précisément cette rigueur qui amène de plus en plus souvent les enseignants, comme les étudiants, à se confronter à la difficulté de cerner clairement les domaines de spécialité abordés, délimitation indispensable pour faire de la terminologie un outil d'aide efficace pour la traduction. Cet impératif se heurte à la créativité actuelle des sciences et des techniques, fondée sur le croisement et les interférences de domaines jusqu'ici cloisonnés. L'objectif de cet article est d'analyser les conséquences de ces évolutions sur l'analyse terminologique, en faisant le pari que les difficultés telles qu'elles sont exprimées, et parfois résolues, par nos étudiants, peuvent contribuer à faire avancer la réflexion théorique sur la nature du domaine et, partant, de la langue de spécialité.

2 La question du brouillage des frontières est abordée dans une première partie, à l'aide de quelques exemples récents, pour ensuite considérer ses conséquences pour la notion de domaine, de langue de spécialité et finalement, pour l'approche terminologique d'un domaine. Trois études de cas permettent d'analyser la manière dont les étudiants abordent cette question, et surtout de mieux comprendre les techniques qu'ils mettent en œuvre pour résoudre les problèmes posés par la délimitation indispensable de frontières. Leurs travaux fournissent les éléments nécessaires à la formulation de quelques propositions pour une approche renouvelée de la notion de domaine en 
terminologie et en langue de spécialité. Sont enfin envisagées les conséquences de ces propositions quant à l'enseignement de la terminologie et à son application à la traduction spécialisée.

\section{Frontières et domaines : une constante transgression}

\subsection{Des problématiques émergentes, qui débordent les frontières reconnues}

3 La terminologie est née au $\mathrm{XX}^{\mathrm{e}}$ siècle du besoin techno-scientifique et économique de fixer des connaissances et des savoir-faire afin de permettre à l'industrie et à la science les échanges indispensables au développement économique à l'échelle de la planète (Rey 1979 : 12-14). Cette clarification, aux objectifs pragmatiques et inter-linguistiques, avait pour cadre un monde qui disposait encore de catégories relativement figées, héritières des classifications scientifiques du XXVIII ${ }^{e}$ siècle et bibliothéconomiques du $\mathrm{XIX}^{\mathrm{e}}$ siècle. Le passage de la révolution industrielle à l'ère numérique au $\mathrm{XX}^{\mathrm{e}}$ siècle $\mathrm{a}$ entraîné le passage du monde des techniques au monde de l'information. La puissance du numérique est notamment fondée sur sa capacité à établir des liens entre des connaissances, des techniques et des savoir-faire jusque-là strictement cloisonnés. Son aptitude à dématérialiser les objets créés a libéré une capacité d'innovation technologique mais aussi économique dont les conséquences apparaissent souvent difficiles à maîtriser, tant sur le plan de leur conceptualisation, que sur celui de leur impact sur le monde réel. Ainsi, les nanotechnologies combinent physique, mathématiques et sciences des matériaux pour produire des applications qui révolutionnent, entre autres, le domaine de la médecine et de la cosmétique, avec la création par exemple des " textiles intelligents », capables de s'adapter aux besoins du corps humain. La révolution de l'information a mobilisé électronique, informatique et tout le domaine des sciences du langage pour dématérialiser l'information et en permettre le partage à l'échelle planétaire, avec des conséquences politiques et économiques incalculables.

4 De ce vaste maelstrom, naissent sans cesse ce que les réseaux sociaux appellent des "bulles de savoir», qui sont autant de problématiques émergentes, telles que l'obsolescence programmée, le droit à l'oubli numérique, l'impression 3D, l'Internet des objets.

5 La structure de ces nouveaux termes, souvent fondée sur des notions contradictoires, reflète ces croisements de domaines parfois paradoxaux et pourtant hautement productifs. Elle est le reflet d'une tectonique d'où émergent sans cesse de nouvelles problématiques liées à la rencontre entre plusieurs domaines

\subsection{Une évolution constante des domaines de spécialisation}

6 Ces évolutions ont largement affecté le classement des domaines de connaissances et d'expertise. Elles ont certes permis une combinaison productive des disciplines, à l'origine de presque toutes les innovations technologiques récentes (Rosnay \& de Closets 2007). Mais, ce faisant, elles en ont brouillé les frontières, rendant problématiques les classements jusqu'alors admis, et générant par exemple dans le 
domaine de la recherche scientifique des comportements contradictoires qui allient une invocation quasi incantatoire de la pluri- (ou multi, ou inter-) disciplinarité avec une défense institutionnelle des prés carrés non moins vigoureuse, notamment sur le plan du financement de la recherche. Dans le monde des bibliothèques, les classifications décimales universelles, qui distinguent nettement sciences, arts et techniques sont battues en brèche par les possibilités multiples qu'ouvrent la capacité d'indexation des bases de données et leurs champs d'interrogation combinables.

7 Par ailleurs, la révolution de l'information, en permettant théoriquement à tous, non seulement l'accès à la réception de l'information, mais aussi à sa production, a profondément transformé les critères qui définissent les notions mêmes de spécialité et d'expertise. On a vu apparaître des communautés d'experts (Beacco 1995; Lave \& Wenger 1991), que ne détermine que la reconnaissance de leurs pairs, sans validation institutionnelle ou académique, et qui font pourtant autorité, évolution favorisée par les nouveaux lieux d'expression que sont les réseaux sociaux, notamment. Ce phénomène affecte particulièrement des domaines de spécialité en pleine transformation, tels que l'informatique, ou l'écologie ${ }^{1}$. C'est toute la notion de spécialisation qui se trouve transformée par ces évolutions permanentes, ce qui ne peut qu'affecter profondément la définition de ce que sont les langues de spécialité et les principes qui régissent leur approche terminologique (Petit $2010: 23$ ).

\subsection{Un défi pour l'appréhension de la notion de langue de spécialité}

8 La raison d'être de l'approche de la langue par le biais du spécialisé est l'affirmation de l'existence, à côté et à partir de la langue générale, de discours dont la caractéristique est d'être l'apanage de communautés définies par un savoir, un métier, des connaissances communes. Ces savoirs partagés donnent naissance à un usage spécifique de la langue générale et à une terminologie adaptée à des besoins de communication particuliers (Percebois 2006).

Or, tout en évoluant extrêmement rapidement, ces ensembles de connaissances débordent actuellement les communautés qui les produisent pour se combiner et se croiser, formant de nouveaux domaines, dont les contours sont bien difficiles à délimiter, remettant en cause toute typologie fixe des communautés qui produisent les discours spécialisés.

10 L'approche de la langue de spécialité se trouve ainsi face à un paradoxe : alors qu'on assiste à une explosion des savoirs, donc des besoins en communication spécialisée, l'impossibilité d'un classement efficace des nouveaux savoirs rend la description et l'analyse de leur mode d'expression de plus en plus problématique. Il convient de noter également que, en raison du rôle dominant de l'anglais dans la circulation des savoirs et des techniques, l'anglais de spécialité se trouve au premier titre concerné, entraînant à sa suite l'ensemble des langues, confrontées à la nécessité d'innovations linguistiques constantes, dans une cascade de néologismes, de calques et d'emprunts divers.

\subsection{Conséquences sur l'approche terminologique du domaine de spécialité}

11 Si ce besoin d'innovation n'est en rien un phénomène nouveau pour l'approche terminologique des langues de spécialité (Humbley 2006), en revanche, la créativité 
terminologique en matière de néonymie s'appuie sur une délimitation précise des domaines pour lesquels valent ces nouveaux termes.

12 La notion de "domaine» occupe en effet une place centrale pour la terminologie, comme le résume Alain Rey (1979: 52) :

[L'objet terminologique] est constitué par les vocabulaires liés à un domaine distinct organisé ou considéré comme organisable, défini thématiquement et socialement; chaque domaine correspondant à la fois à un contenu (critère sémantique) et à des pratiques réglées (critère socio-culturel).

C'est bien ce concept de « pratiques réglées » qui se trouve actuellement fragilisé. Or, si les linguistes s'accordent sur la place centrale du «domaine » en terminologie, la notion même est rarement explicitement définie. La terminologie utilisée pour désigner le domaine fait l'objet de nombreuses variations: domaine notionnel ou d'activité (Rey 1979: 83; Petit 2010:23), domaine de connaissance, d'activité et de discours (Bessé 2000 : 183-185), domaine d'exploitation (De Vecchi $2009: 2$ ), « practical social domain" (Rey \& Sager 1995 : 109) ; le domaine terminologique semble dans tous les cas porter la charge de l'interrelation entre savoir et connaissances d'une part, action, pratique et transformation du monde d'autre part.

Ainsi, Margaret Rogers, après avoir intitulé sa conférence plénière donnée lors du $34^{\mathrm{e}}$ colloque du GERAS: "What is a domain? and is this a useful question?", propose la définition suivante: " a set of interrelated social constructs (to help us) interact with the world".

Or, la notion de domaine est la pierre de touche de la terminologie dans la mesure où, non seulement elle définit son champ d'application, mais aussi elle constitue le critère qui permet de mesurer la cohérence des définitions.

Porteur de la visée pragmatique externe de la terminologie, le domaine l'est aussi de sa cohérence interne. Pour Bruno de Bessé $(2000$ : 183) :

Le domaine est un constituant du concept: l'ensemble concept-définition est incomplet et donne l'impression d'être un peu bancal. [...] Le concept, sa définition (et son terme) appartiennent obligatoirement à un domaine. [...] Le domaine permet d'indiquer le système conceptuel auquel appartient le concept.

17 Autrement dit, les deux concepts-clés de la terminologie, le terme et sa définition, ne prennent leur sens que si l'on est capable de les relier à un domaine spécifique clairement déterminé. Mais rien n'indique comment le domaine lui-même peut être défini, comme en témoigne cette définition de Teresa Cabré :

L'ensemble des termes d'un domaine, c'est-à-dire sa terminologie, reflète la structure conceptuelle du sujet et chacun des termes dénomme un concept du réseau structuré du domaine en question. $(1998: 147)$

18 Si ces deux auteurs de référence se rejoignent sur la notion de domaine comme fondant la cohérence terminologique, on ne peut se défendre d'une certaine impression de circularité : le terme est défini par le domaine, mais le domaine est défini par l'ensemble des termes.

19 L'impression est confirmée par cette proposition définitoire de l'office québécois de la langue française :

Domaine

Le domaine, qui constitue une branche spécialisée de la connaissance, sert de cadre à l'intérieur duquel est établi le champ conceptuel. Il correspond, d'une certaine façon, à une orientation que l'on attribue à la définition. Il est donc important de 
définir un concept en fonction du domaine ou du sous-domaine dans lequel il

s'inscrit. (Bedard \& Darras 2009 : 8) problématiques à la croisée de plusieurs domaines de connaissances et d'activité sans brouiller les pistes et introduire une confusion dans les critères terminologiques classiques et leur cohérence dans une perspective onomasiologique. L'exemple de l'«obsolescence programmée », concept encore obscur quelques mois avant de devenir l'objet d'une loi et d'un débat parlementaire, est significatif. Derrière cette formule étrange se cache une bascule de point de vue emblématique de ce début du XXI ${ }^{\mathrm{e}}$ siècle : nous sommes passés en quelques mois de la gestion (ou management) de l'obsolescence à la mise en cause de sa programmation. Plus exactement, une question technique (gérer l'obsolescence des produits, notamment électroniques) est devenue une question économique et politique (lutter contre une obsolescence délibérément programmée pour stimuler la consommation). Le terme " obsolescence programmée » recouvre deux domaines distincts, l'un managérial, l'autre politique, qui traitent cependant bien du même phénomène, de deux points de vue différents, voire opposés. 

plusieurs couches successives de savoirs et de visions du monde réel (Gambier 1987). C'est le cas du terme "droit à l'oubli numérique », construit sur l'emboitement contre nature des connotations positives de «droit à " et négative de «oubli ». Le terme «numérique » est lui souvent associé au syntagme «mémoire numérique ». C'est ainsi que le syntagme complexe «droit à l'oubli numérique » produit une double impression d'étrangeté, en rapprochant la notion de « droit » de celle d'« oubli », puis en créant le terme inattendu d'« oubli numérique ». Ce faisant, ce néologisme fait appel au domaine législatif qui définit les droits de l'individu, à celui de l'histoire et de la conservation des traces et à celui de l'information et de sa diffusion.

La traduction d'un texte portant sur ces nouveaux concepts exige ainsi la mise en œuvre de ressources multiples, comprenant des termes qui peuvent relever d'un seul ou de plusieurs des domaines concernés. Le traitement terminologique de ces termes doit prendre en compte cette complexité afin de produire des ressources terminologiques adaptées et directement utilisables pour le traducteur. Ceci implique le renoncement à la logique positiviste de l'univocité du rapport entre terme et concept, et l'adoption d'une position qui privilégie le besoin de communication. Alain Rey décrit ainsi les risques de stérilité inhérents à une position normative, tentée d'imposer au réel un ordre théorique :

Le positivisme rigide qui gouverne souvent la théorie terminologique est probablement une étape nécessaire, au moment de sa constitution. Mais le décalage entre la volonté de description et la méconnaissance des objets à décrire condamnerait cette activité à l'impuissance. (Rey 1979 : 15)

\subsection{Repenser les outils terminologiques}

Cette complexité et le flou qui en résulte pour la définition du domaine affectent l'ensemble des concepts-clés de la terminologie, concepts qui en font pourtant un outil de description à la fois exigeant et efficace. "Étude des systèmes notionnels qui structurent un domaine de spécialité » (Cabré 1998: 48), la méthode terminologique s'appuie sur la sélection des termes pertinents, leur définition en contexte, puis la représentation hiérarchique des liens sémantiques qui relient ces termes dans le domaine considéré.

$\mathrm{Au}$ « centre du problème terminologique » (Rey 1979: 39), la définition du terme doit exprimer les limites du concept en décrivant les caractéristiques qui l'inscrivent dans un domaine de connaissances. Les caractéristiques énoncées dans cette définition doivent pouvoir être représentées graphiquement sous forme d'une arborescence de liens sémantiques qui, en définissant le statut de chaque terme, le relie aux autres, transformant ainsi des informations éparses en l'image conceptuelle d'un domaine de connaissances. Or, comme le signalait déjà Alain Rey (1979 : 43) :

Si le domaine n'est pas déterminé et construit, ou encore s'il s'agit d'un domaine hétérogène, pragmatique et empirique, la définition terminologique pure est absolument impraticable.

\subsection{Revenir à la vocation initiale de la terminologie ?}

En posant le cadre d'interprétation, le domaine fixe la forme du concept et permet de le classer en le reliant aux autres termes, selon une logique soit hiérarchique 
(hyperonymes, hyponymes, isonymes), soit concrète (cause/conséquence, spatiale, temporelle). La nécessité de traiter de sujets qui recouvrent plusieurs domaines déstabilise donc une logique avant tout positiviste et conçue pour éliminer toute ambiguïté. Une autre voie s'ouvre qui ramène à une vision $d u$ «terminologue intermédiaire, spécialiste de communication, interface entre les spécialités, les sciences cognitives, la documentation et l'expression linguistique " (Humbley 1998: 3). Le domaine devient un concept pragmatique et dynamique, «résultat de la dialectique entre l'intégration (interdisciplinaire) et la parcellisation (hyperspécialisation)» (Gambier 1991 : 37).

Dans cette conception, la vocation du terminologue est de permettre le transfert des connaissances, de spécialiste à spécialiste, de spécialiste à non-spécialiste et d'une langue à l'autre. Si ce transfert implique toujours une délimitation des termes par leur définition, il met surtout l'accent sur l'usage du terme, dans une conception fonctionnaliste du discours, qui établit le lien avec les objectifs de la communication. D'une terminologie qui fixe les connaissances, on passe à une "terminologie d'interface » que Catherine Resche présente ainsi :

Designating objects, tools or concepts via a rigorous denomination in order to establish and unify the terminology of a specialised field is one thing. Deciding which terms will be most helpful for a given public with specific purposes is another. (Resche $2000: 630$ )

Le domaine ne relève plus d'une classification préexistante mais doit être déterminé dans une perspective de besoins spécifiques de communication, liés à une activité humaine susceptible d'évolutions. Pour stimulante que soit cette proposition, elle ne résout pas pour autant la question posée par l'effacement des frontières des domaines, notamment dans le cas de l'approche terminologique de la traduction spécialisée. La traduction d'un texte spécialisé exige en effet une parfaite maîtrise du réseau conceptuel du ou des domaines abordés par le texte (Rey 1979 : 60). L'appréhension de leur nature et de leurs frontières est indispensable pour une traduction qui respecte les objectifs de communication initiaux. Cette découverte du domaine est le produit d'une interaction constante entre terminologie et traduction, interaction mise en évidence par les tâtonnements des étudiants traducteurs, dans leur démarche d'élaboration des ressources terminologiques indispensables pour étayer une traduction raisonnée.

\section{S'appuyer sur l'application didactique pour faire évoluer la perspective théorique}

31 Des travaux réalisés par des étudiants en master de traduction spécialisée offrent un terrain d'observation privilégié de la manière dont l'apprenti traducteur construit, pour son projet de traduction, sa propre représentation de ce qu'est un domaine terminologique. "Construit» est bien le terme qui convient, car la question de la nature du domaine cristallise au long de l'année à la fois les inquiétudes et les plaisirs de la découverte linguistique. 


\subsection{Réaliser un dictionnaire spécialisé bilingue comme appui à une traduction spécialisée} documentaire, d'analyse de corpus, de terminologie, de bases de données et de traductologie. Chacun des enseignements est conçu pour fournir les bases théoriques indispensables mais aussi les techniques et outils adaptés à la réalisation du mémoire. À partir du sujet choisi et/ou du texte à traduire, l'étudiant constitue des corpus spécialisés et de vulgarisation qui lui permettent d'explorer le ou les domaines qu'il doit aborder. Ces corpus sont analysés notamment grâce aux outils de la linguistique de corpus afin de fournir le matériau linguistique et conceptuel nécessaire au traitement terminologique (Kübler \& Aston 2010). L'initiation aux bases de données permet une compréhension de la logique d'interrogation combinée de champs dans la multibase terminologique que constitue Artes. Le travail de traduction s'appuie à la fois sur l'ensemble des techniques abordées lors des divers cours de traduction et sur les ressources terminologiques constituées dans le cadre du mémoire. Les combinaisons de langues sont multiples, la plus fréquente étant néanmoins, et de manière prévisible, la traduction de l'anglais vers le français.

\subsection{Place de la notion de domaine dans le processus de recherche}

L'une des premières difficultés auxquelles est confronté l'étudiant est l'alternative entre la priorité donnée à la sélection du texte et celle donnée à la recherche d'un sujet potentiellement productif en matière de discours spécialisés. Les exigences posées quant au choix du texte ${ }^{4}$ amènent souvent à commencer par cette étape pour ensuite définir le sujet traité. C'est alors que se pose la difficile question de la détermination du domaine terminologique, préalable qui paraît indispensable pour la sélection des termes du dictionnaire. S'agissant de textes récents, la plupart des textes, quels que soient la discipline ou le domaine concernés, se structurent autour de problématiques

ASp, $64 \mid 2013$ 
complexes qui mobilisent des savoirs variés. Cette première découverte déconcerte des étudiants pour qui la spécialisation implique le traitement d'un sujet précis, dont ils présupposent qu'il est délimité. La difficulté n'est pas moindre pour ceux qui ont choisi d'abord un sujet avant de rechercher un texte. Les premières recherches de corpus suscitent la même perplexité : chaque sujet, aussi pointu semble-t-il, recouvre une sorte de millefeuilles de domaines de connaissances et d'activités. Comment choisir entre ce double impératif : prendre en compte la complexité du texte et du sujet, tout en définissant un cadre conceptuel délimité qui seul permettra une sélection de termes cohérente et adaptée à la démarche terminologique ? La piste de la distinction entre micro- et macro-domaines ne résout pas le problème car elle implique une hiérarchisation des domaines qui ne correspond pas à la réalité des croisements de savoirs rencontrés.

L'exemple de l'« obsolescence programmée ", sujet proposé par une étudiante en début d'année, offre une illustration de ces difficultés. Après quelques semaines de recherche de corpus, l'étudiante indique se heurter à une relative pauvreté des ressources spécialisées, les textes collectés représentant le plus souvent un point de vue militant et vulgarisateur. Une discussion sur les termes utilisés pour la recherche met au jour le fait que le syntagme " obsolescence programmée » prédétermine un type de discours et que le simple fait d'enlever les guillemets de l'équation de recherche permet d'avoir accès à un tout autre gisement d'informations, celui de l'obsolescence et de son management. Le choix du terme " obsolescence programmée » relève en effet déjà d'un contre-discours. L'étudiant qui, s'agissant de domaines très spécifiques, s'attend à une univocité de discours, est confronté au fait que ces domaines sont en réalité le lieu de l'expression de voix multiples qui se répondent et s'affrontent. Cette découverte va débloquer la situation. La prise en compte de la diversité des mots clés et de leurs liens avec des points de vue divergents permet à la fois de rassembler un corpus représentatif et de définir progressivement un cadre d'analyse terminologique. Les études de cas qui suivent permettent d'analyser et d'illustrer ce processus d'élaboration intellectuelle.

\section{4. Études de cas à partir de productions d'étudiants}

37 Les analyses incluses dans le mémoire réalisé par les étudiants portent sur le processus de recherche documentaire, les choix terminologiques et les difficultés de traduction. Fruits d'une réflexion menée sur une année, elles offrent un panorama intéressant des problèmes rencontrés et des réponses qui ont été apportées. Il peut bien sûr s'agir de difficultés pratiques, mais leur résolution implique des choix qui reposent sur une démarche intellectuelle constituée au cours du travail de recherche. C'est en cela que ces travaux paraissent pouvoir alimenter une réflexion sur les fondements théoriques de la terminologie et ses liens avec la traduction.

\subsection{De l'analyse d'un texte à la conceptualisation d'un domaine}

L'étude de cas $n^{\circ} 1$ s'appuie sur un extrait d'un protocole de recherche présenté par une étudiante ${ }^{5}$ dont le texte avait pour sujet le « processus d'injection des aérosols dans la stratosphère ». 
Pour tenter d'analyser le cadre dans lequel s'insère ce sujet, l'étudiante applique à son corpus et son texte une technique d'analyse proposée en cours, à savoir «PositionProblem-Solution" ou "PPS », technique anglo-saxonne d'analyse de problème (Hoey 1994 : 26-45). En voici le résultat tel que le présente l'étudiante :

Position : nous faisons face aujourd'hui au réchauffement climatique

Problem: l'équilibre énergétique mondial est perturbé par l'effet de serre additionnel

Solution : la géoingénierie utilise les aérosols pour atténuer ces effets de serre

Elle en tire les conclusions suivantes:

Je connaissais déjà le phénomène d'effet de serre, je me suis donc demandé ce qu'était la géoingénierie, pourquoi elle s'intéressait aux aérosols, et en quoi ceux-ci pouvaient selon certains scientifiques contribuer à diminuer le réchauffement climatique. J'avais trouvé mon sujet: "La géoingénierie et les aérosols» qui a évolué par la suite pour devenir: "les aérosols et leur rôle dans l'équilibre énergétique mondial ».

La première étape (le PPS) a permis d'élargir le cadre de la simple description d'une technique à la problématique générale dans laquelle elle s'intègre. Cette problématisation se fait notamment par la découverte du néologisme " géoingénierie " qui introduit une dimension à la fois technique et environnementale. Ce terme pivot permet de parvenir à l'étape finale de conceptualisation du domaine, qui le situe nettement dans une perspective alliant ressources énergétiques et gestion mondiale de l'environnement. Le travail de conceptualisation s'appuie ici sur l'analyse du sujet initial pour l'insérer dans un domaine plus global qui va permettre à son tour de définir un cadre d'analyse terminologique.

\subsection{Aborder un domaine complexe avec les outils de l'analyse de corpus et de la terminologie}

41 L'étude de cas $n^{\circ} 2$ concerne un travail en cours de réalisation ${ }^{6}$. Les éléments analysés ci-dessous ont été donnés lors de la pré-soutenance qui intervient à mi-parcours et permet aux étudiants, en formalisant les acquis, d'identifier les pistes qui doivent encore être creusées. Ils concernent d'abord l'analyse des sources (figures 1 et 2), puis les évolutions de la représentation graphique du domaine (figures 3 et 4 ).

Les deux premiers schémas illustrent la place de l'analyse comparative des sources pour le traitement terminologique d'un sujet complexe : le «trafic des diamants du sang ». La représentation en est donnée dans les figures 1 et 2. 
Figure 1 Analyse du corpus français

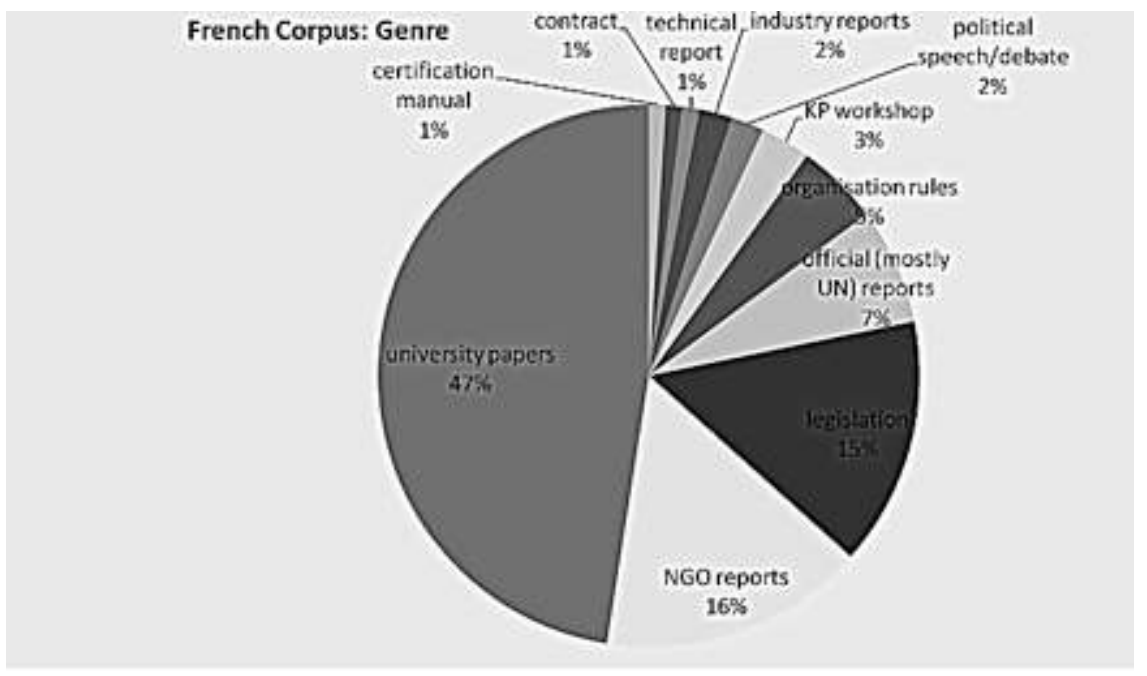

Source : version de pré-soutenance d'un mémoire de terminologie de master 2

Figure 2 Analyse des sources en anglais

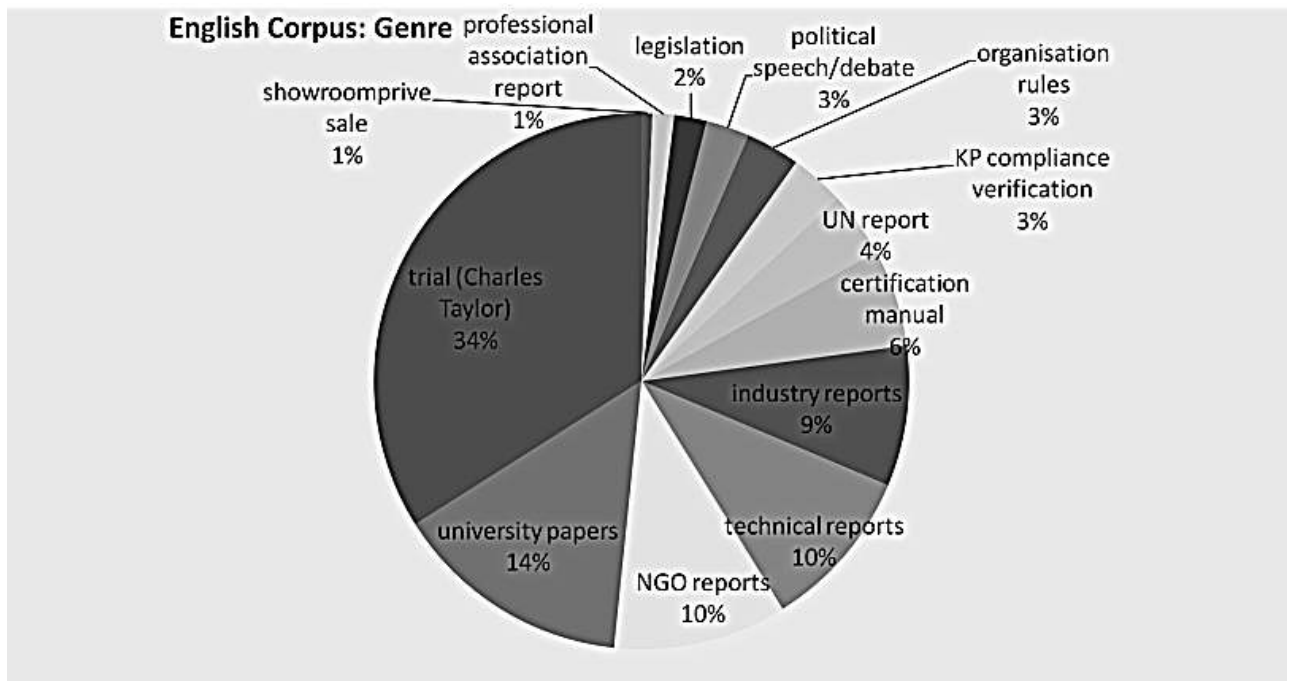

Source : version de pré-soutenance d'un mémoire de terminologie de master 2 techniques, écrits universitaires notamment. Néanmoins, la proportion occupée par ce dernier type d'écrits est beaucoup moins élevée en anglais qu'en français, non faute de ressources disponibles, mais en raison de la place considérable (34\%) prise par les minutes du procès de Charles Taylor. Cet ex-chef de l'état du Liberia, impliqué au Liberia et au Sierra-Leone dans le trafic de diamants, a en effet été jugé pour crimes de guerre en 2012 aux États-Unis. Ce procès a eu un large retentissement, d'où l'importance des publications qu'il a généré.

Il est clair que cet écart dans la typologie des sources va induire des disparités dans la nature des termes utilisés et dans leur définition: ces disparités sont l'objet même de l'analyse contrastive préalable au travail de traduction, et devront être prises en compte dans la sélection des termes et leur traitement. 
La comparaison entre le troisième et le quatrième schéma (figures 3 et 4 ) donne un aperçu de la manière dont évolue la représentation du domaine concerné, au fur et à mesure que progressent les recherches menées pour la constitution de ressources terminologiques.

Le schéma 3 propose une première représentation graphique de l'organisation des termes sélectionnés comme représentatifs du domaine analysé. Ce schéma a été soumis au bout de deux mois de recherche. Il met en évidence les regroupements thématiques et non les termes pris isolément.

Figure 3 Représentation initiale du domaine

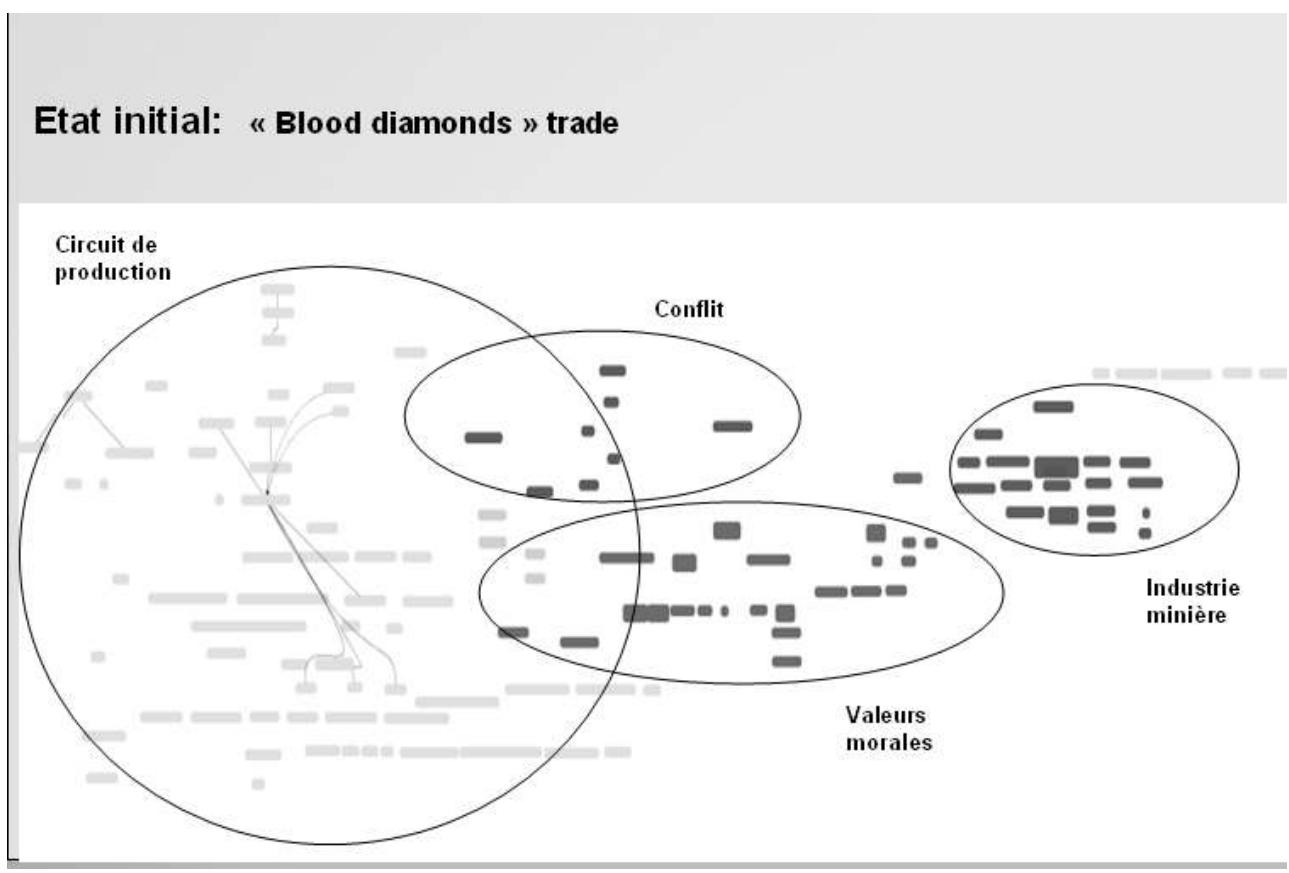

Dans un premier temps, l'intitulé choisi pour le domaine est "blood diamonds trade». L'ébauche d'arborescence fait apparaître quatre grands ensembles de termes: « circuit de la production ", « conflit ", « valeurs morales » et « industrie minière ».

Le schéma 4 fournit la représentation graphique de l'organisation des termes présentée lors de la pré-soutenance, à mi-parcours de cette année de master 2 , au bout de six mois de recherche. Ici aussi, on met en évidence les regroupements thématiques, et non les termes. 
Figure 4 Représentation du domaine lors de la pré-soutenance ${ }^{7}$

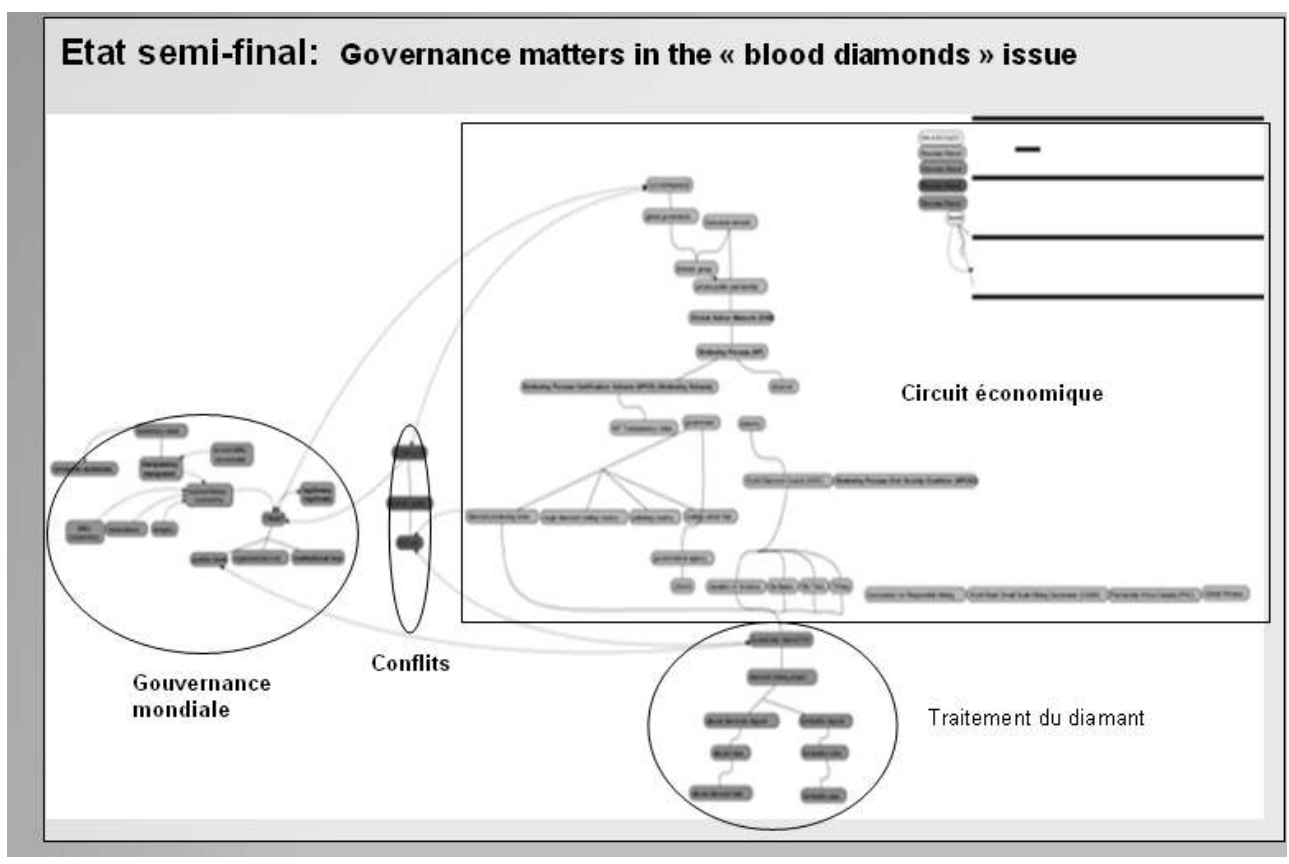

La nature des ensembles a changé, leur représentation dans l'espace aussi. L'ensemble « gouvernance mondiale » est apparu, ce qui se manifeste par un changement d'intitulé du domaine, avec le remplacement de «trade » par « issue ». La position des ensembles indique clairement le point de vue selon lequel la gouvernance mondiale régit, via la gestion des conflits, les relations entre les deux autres ensembles, à savoir le traitement du diamant et son circuit économique. Le domaine ne se situe plus dans la sphère des échanges économiques, mais dans celle de la moralisation des échanges économiques mondiaux.

Ce changement de point de vue sur la nature du domaine a des conséquences sur le traitement terminologique, comme en témoigne le courrier suivant, postérieur à la présoutenance, et adressé par l'étudiante à sa directrice de mémoire :

J'ai travaillé sur les liens entre «blood diamonds» et « conflict diamonds», puis sur l'équivalent français. Deux problèmes se posent :

- «blood diamonds» et « conflict diamonds » sont employés comme des synonymes par la communauté de discours. Néanmoins, l'étude des sèmes dans les contextes définitoires de "blood diamonds" montre que la notion diffère de celle de « conflict diamonds » telle qu'elle est définie par les organisations internationales. «blood diamonds » n'a pas de définition officielle, au contraire.

- l'équivalent "diamants du sang» de "blood diamonds", utilisé dans les traductions du corpus parallèle, n'a pas exactement la même connotation. Les contextes sont très différents d'une langue à l'autre. Je pressens donc que "diamants du sang", s'il recouvre la même réalité que "blood diamonds », ne recouvre pas les mêmes problématiques. L'anglais l'associe clairement à tous les diamants extraits dans des contextes de violence. Le français, quant à lui, est plus critique face à la notion, qui est présentée autant comme un terme de politique que comme une notion marketing. S'agit-il d'un défaut du corpus ou d'une réalité ? Cela me pose problème pour rédiger la définition de « diamants du sang ».

Quel est votre avis sur ces deux questions?

51 L'analyse de l'étudiante et son questionnement montrent qu'elle fait le lien entre l'analyse du corpus collecté, la diversité des sources représentatives du domaine et la 
valeur sémantique que prennent les termes. L'utilisation de l'expression « défaut du corpus » met en évidence la volonté de construire un corpus qui représente l'ensemble des types de discours et des communautés de discours concernés. La définition est conçue comme la description de la valeur que prend le terme dans une communauté de discours spécifique.

\subsection{De l'analyse de domaine aux choix de traduction}

Dans une troisième et dernière étude de cas, l'auteur du mémoire montre comment cette compréhension de la problématique du domaine abordé fonde les choix de traduction. Il s'agit d'un travail réalisé en 2011-2012 et qui a fait l'objet d'une publication ${ }^{8}$ l'année suivante. L'analyse de l'étudiant porte sur la traduction du terme " sustainable development " dans le cadre d'un dictionnaire terminologique portant sur l'« économie écologique ».

53 Après avoir attiré l'attention sur le fait que les économistes écologiques français refusent de traduire l'espagnol « sostenible » et l'anglais « sustainable » par « durable », il explique qu'en effet «ils remettent en question la pérennité illusoire de l'exploitation des ressources que sous-tend la notion de 'développement durable'». Par conséquent, le traducteur francophone, selon qu'il choisit le terme "durable " ou "soutenable", marque ou non une adhésion aux représentations de l'économie écologique. Le choix du domaine et le point de vue qu'il implique va affecter non seulement la traduction du terme mais aussi ses collocations. Dans le domaine traité, les collocations de «soutenable» seront ainsi «soutenable socialement", "futur soutenable», " décroissance soutenable».

Ce dernier exemple met en évidence la manière dont le contexte collocationnel confère à un terme sa valeur sémantique, spécifique à un domaine et, au-delà, à une problématique de l'activité humaine. Il montre ainsi le lien entre délimitation du domaine et analyse terminologique comme préalables à une traduction raisonnée.

\section{Quelle place pour la notion de domaine dans l'approche terminologique de la langue de spécialité ?}

55 Les travaux présentés dans la section 4 , les difficultés qu'ils mettent en lumière, ainsi que les réponses qu'ils apportent, ouvrent plusieurs pistes de réflexion pour une évolution de la méthodologie terminologique appliquée à la traduction, et plus particulièrement pour la question décisive du rôle de l'identification du domaine de spécialité dans l'analyse terminologique.

\subsection{Le discours d'une ou plusieurs communautés autour d'une problématique centrale}

La complexification de l'organisation des connaissances humaines et des activités qui en découlent amène à revoir la notion de domaine de spécialité qui cesse d'être monolithique, comme le souligne Michel Petit $(2010: 23)$ :

Un domaine spécialisé ne se réduit ni à la seule activité de ses acteurs emblématiques ni, a fortiori, à leur seule activité de discours, voire à leur seule 
activité de discours de recherche, mais constitue un système essentiellement complexe, dont l'identité en tant que domaine spécialisé résulte de la combinaison spécifique de l'ensemble des éléments d'ordre divers qui lui sont rapportés. projet de traduction, la constitution d'un corpus spécialisé comparable et son analyse permettent de cerner une problématique centrale (par ex.: «comment moraliser la circulation internationale des diamants») puis d'identifier l'ensemble des communautés de discours qui sont concernées par ces enjeux. Le critère de sélection des termes pertinents n'est plus l'appartenance à un domaine de savoir ou même d'activité mais une analyse des besoins de communication de diverses communautés. Celles-ci partagent des enjeux mais leurs intérêts peuvent s'affronter. Le domaine est "une synergie constante d'usages différents, de logiques parfois contradictoires » (Gambier 1991 : 39).

\subsection{D'une logique de frontières à une logique de questionnement}

Le domaine de spécialité n'apparait plus comme un territoire clos mais plutôt comme une constellation de productions centrées autour d'enjeux et de questionnements communs.

La terminologie passe d'une logique positiviste descriptive, qui vise à fixer une réalité en la nommant, à une logique fonctionnaliste et pragmatique, qui propose des ressources linguistiques adaptées à des besoins de communication spécifiques. En cela, elle rejoint l'affirmation de M. A. K. Halliday (1991: 39): « Here the organizing concept is not the object that is being investigated - the field of study - but the kinds of questions that are being asked ».

Le "paysage » terminologique évolue alors en fonction du regard que portent sur lui les membres des communautés concernées. C'est cette approche dynamique et multifacettes que prônent déjà dans leur étude des "tribus académiques" les sociolinguistes T. Becher et P. R. Trowler $(2001: 16)$ :

The relationship between the identifiable shifts in the landscape on the one hand and academic cultures, work conditions and disciplinary communities on the other is dynamic, complex and far from tightly-coupled. The landscape metaphor turns out to be a particularly appropriate one: land exists without an observer, but landscape does not: the 'landscape' is the projection of human consciousness, the way the land is perceived and responded to.

\subsection{Redéfinir des concepts opératoires pour l'appréhension d'un nouveau domaine de connaissances}

61 Le risque de la prise en compte de la complexité dans la détermination d'un domaine et des termes qui en relèvent est de diluer les critères d'analyse qui font la force de l'approche terminologique et, notamment, le rapport univoque fondateur entre «terme » et « concept ». Si tout est affaire de point de vue, toutes les définitions se valent, et seule compte l'analyse sociolinguistique de la communauté de discours (Slodzian 2006). Serait alors perdue la capacité spécifique de l'approche terminologique qui, en mettant au jour les liens qui unissent et opposent les termes, dessine et structure un paysage de l'activité humaine. 
62 Les propositions énoncées ci-dessus doivent être envisagées dans leur application concrète à la grille d'analyse terminologique. Ce glissement de point de vue affecte d'abord la définition de ce qu'est un terme, considéré non plus seulement du point de vue de sa valeur dénotative mais aussi de sa valeur sémantique en contexte, donc de sa valeur connotative. S'ensuit également une adaptation de la notion de définition, qui ne se situe plus dans un univers hiérarchisé, et où les caractéristiques de chaque terme ne le définissent plus seulement comme appartenant à un ensemble plus large. Le terme peut être défini par la fonction spécifique qu'occupe le concept désigné dans une communauté de discours et par rapport à une intention de communication, c'est-à-dire par rapport à un genre et une culture. Cette conception du terme et de sa définition est proche de celle de John Humbley (2006: 102), pour qui il convient de justifier la notion de synonymie en terminologie en proposant de conserver «des synonymes qui permettent un rattachement symbolique à une autre communauté ». Cette vision du terme en fait, selon l'expression de F. Rastier (2010: 101) «a semantic form, profiled against a background». Et c'est cette forme sémantique qui détermine le registre des collocations du terme dans un domaine donné, comme l'a montré la troisième étude de cas à propos de l'«économie politique » (cf. 4.3). Enfin, l'arborescence terminologique qui dessine dans l'espace les liens entre les termes abandonne sa verticalité hiérarchique pour prendre en compte la circularité caractéristique de nombreux domaines, dans une représentation du monde qui doit plus à l'analyse systémique qu'aux classifications hiérarchisées du XIX ${ }^{\mathrm{e}}$ siècle.

63 Ce n'est certainement pas un hasard si cette nouvelle approche est largement facilitée par les évolutions technologiques, de deux manières. D'une part, les outils de collecte et d'analyse de corpus permettent maintenant une analyse fine des types de sources indispensables à toute étude terminologique visant à prendre en compte la diversité des communautés de discours. D'autre part, les bases de données, en multipliant les possibilités de combinaison des champs d'interrogation, fournissent toute la souplesse nécessaire à une approche multi-facette du terme et de sa valeur.

\section{Conclusion}

\section{Valeur heuristique de la notion de domaine pour l'enseignement de la terminologie}

Dans le cadre de l'enseignement de la terminologie appliquée à la traduction, il apparaît clairement que la notion de domaine de spécialité doit être présentée non comme un préalable mais comme un objectif. Il ne s'agit pas de commencer par choisir un domaine de spécialité puis de traiter les termes qui le caractérisent, mais de découvrir derrière un sujet spécialisé une problématique plus vaste, incluant ses implications sociales, économiques ou politiques. Loin de se limiter à la constitution d'un glossaire, le travail de recherche terminologique de l'étudiant consiste à mettre au jour, à travers sa terminologie, un pan de connaissances lié à l'activité humaine, avec ses enjeux, ses conflits, ses évolutions et ses contradictions, tels que les termes et leur phraséologie les expriment dans les deux langues et les deux cultures comparées. Définitions, collocations et arborescences fournissent une représentation codifiée de ce domaine, tel qu'il se dessine à la fin de la recherche terminologique, à partir de la collecte et de l'analyse d'un corpus comparable représentatif des productions discursives pertinentes. L'enseignement de la terminologie doit s'appuyer sur 
l'acquisition des compétences nécessaires pour la constitution raisonnée et critique de corpus comparables, ce qui inclut la recherche d'information et les outils d'analyse de corpus.

Une valeur sémantique du domaine déterminée par les conditions de production du discours spécialisé

Il s'agit d'appréhender le discours spécialisé à travers une analyse complète de ses conditions de production et de ses objectifs : la communauté de discours est vue comme un univers qui n'est pas homogène, mais traversé de contradictions, d'enjeux et de conflits, mis en évidence par un traitement terminologique problématisé. C'est cette problématique commune qui détermine les communautés de discours concernées, à travers les enjeux qui les unissent ou les opposent. Ces communautés, à leur tour, déterminent les objets spécialisés tels qu'ils sont présentés dans leurs productions discursives et documentaires, selon les règles et les normes des circuits de communication et des genres qui les caractérisent. La notion de domaine de spécialité, dans cette perspective, devient un concept dynamique et fonctionnel, défini par des besoins de communication évolutifs et éventuellement contradictoires. Elle permet de penser les notions de terme et de définition en prenant en compte la question de la valeur spécifique à ce domaine.

Pour une approche du domaine terminologique adaptée aux besoins de la traduction pragmatique

La discussion de la notion de domaine met en évidence la nécessité d'une approche de la terminologie qui procède des textes eux-mêmes, et revient vers eux, au lieu d'être appréhendée comme une réalité extérieure. (Froeliger à paraître: ch.3). Le texte est considéré comme un élément du réseau des productions discursives représentatives d'un domaine dans sa complexité et dans ses contradictions, puisque "le régime terminologique des textes varie selon les genres et les discours» (Rastier 1995). La multiplicité des valeurs du terme est prise en compte, ainsi que ses implications pour son environnement collocationnel et phraséologique. Cette "terminologie textuelle » (Slodzian 1995) permet de répondre aux besoins de la traduction spécialisée en proposant la constitution de ressources contextualisées, qui reflètent la complexité de la culture-source comme de la culture-cible.

\section{BIBLIOGRAPHIE}

Ahmad, Khurshid, Andrea Davies, Heather Fulford et Margaret Rogers. 1994. « What is a term? The semi-automatic extraction of terms from text ». In Proceedings of the Translation Studies: An Interdiscipline (Selected Proceedings of the Vienna Conference), 267-278.

Beacco, Jean-Claude. 1995. « À propos de la structuration des communautés discursives : beauxarts et appréciatif ». Les Carnets du Cediscor. Publication du Centre de recherches sur la didacticité des discours ordinaires 3, 136-153. <http://cediscor.revues.org/523>. 
Becher, Tony et Paul R. Trowler. 2001. Academic Tribes and Territories. Buckingham : Open University Press.

Bedard, Jean et Xavier Darras. 2009. La rédaction de définitions terminologiques. Montréal : Office Québécois de la Langue Française. <http://www.oqlf.gouv.qc.ca/ressources/bibliotheque/ terminologie/redaction_def_terminologiques_2009.pdf>. Consulté le 5/06/2013.

Bessé, Bruno de. 2000. « Le domaine ». In Béjoint, H. et P. Thoiron (dir.), Le sens en terminologie. Lyon : Presses Universitaires de Lyon, 182-197.

Cabré, Marie Teresa. 1998. La terminologie : théorie, méthode et applications. Ottawa : Presses de l'Université d'Ottawa et Paris : Armand Colin.

De Vecchi, Dardo. 2009. « Pragmaterminologie, une terminologie de l'entreprise en évolution». In Actes de la Ve Journée scientifique REALITER 2009, Milan, 9 juin 2009.

Froeliger, Nicolas. À paraître. Les noces de l'analogique et du numérique. Paris : Belles-Lettres.

Gambier, Yves. 1987. « Problèmes terminologiques des pluies acides : pour une socioterminologie ». Meta: Journal des traducteurs 32/3, 31-58. <http://www.erudit.org/revue/meta/ 1987/v32/n3/002791ar.pdf>. Consulté le 5/06/2013.

Gambier, Yves. 1991. « Travail et vocabulaire spécialisés : prolégomènes à une socioterminologie ». Meta: Journal des traducteurs 36/1, 8-15. <http://www.erudit.org/revue/META/ 1991/v36/n1/002795ar.pdf>. Consulté le 5/06/2013.

Grandjou, Céline et Isabelle Mauz. 2007. « Un « impératif scientifique » pour l'action publique? Analyse d'une compétition pour l'expertise environnementale ». Socio-logos 2 . <http://sociologos.revues.org/893>. Consulté le 17 août 2013.

Halliday, Michael A.K. 1991. «Towards probabilistic interpretation». In Ventola, E. (dir.), Functional and systemic linguistics. Berlin, New York : Walter de Gruyter, 39-61.

Hoey, Michael. 1994. « Signalling in discourse: a functional analysis of a common discourse pattern ». In Coulthard, M. (dir.), Written and Spoken English Advances in Text Analysis Londres : Routledge, 26-45.

Humbley, John. 1998. « Le terminologue et le spécialiste de domaine ». ASp 19-22, 137-149.

Humbley, John. 2004. « La réception de l'œuvre d'Eugen Wüster dans les pays de langue française » <http://halshs.archives-ouvertes.fr/halshs-00276087>. Consulté le 5/06/2013.

Humbley, John. 2006. « La néologie : interface entre ancien et nouveau ». In Greenstein, R. (dir.), Langues et cultures : une histoire d'interface. Paris : Publications de la Sorbonne, 91-103.

Kübler, Natalie et Guy Aston. 2010. « Using corpora in translation ». In Mc Carthy, M. et A. O’Keefe (dir.), The Routledge Handbook of Corpus Linguistics. Londres : Routledge, 505-515

Lave, Jean et Étienne Wenger. 1991. Situated Learning: Legitimate Peripheral Participation. Cambridge : Cambridge University Press.

Millerand, F., L. Heaton et S. Proulx. 2011. «Émergence d'une communauté épistémique : création et partage du savoir botanique en réseau ».<http://www.tela-botanica.org/sites/ botanique/fr/documents/biblio/articles_en_ligne/MillerandHeatonProulx_Tela.pdf>.Consulté le 17 août 2013.

Percebois, Jacqueline. 2006. « Les communautés discursives à l'interface du linguistique et du disciplinaire ». In Greenstein, R. (dir), Langues et cultures : une histoire d'interface. Paris : Publications de la Sorbonne, 67-89. 
Petit, Michel. 2010. « Le discours spécialisé et le spécialisé du discours : repères pour l'analyse du discours en anglais de spécialité ». E-rea 8/1 <http://erea.revues.org/1400>. Consulté le $5 / 06 / 2013$

Rastier, François. 1995. « Le terme : entre ontologie et linguistique ». La Banque des mots 7, 35-65. Rastier, François. 2010. "Web semantics versus the semantic web? The problem of keyness ». In Bondi, M. et M. Scott (dir.), Keyness in texts. Amsterdam, Philadelphie : John Benjamins Publishing, 93-110.

Rosnay de, Joël et François de Closets. 2007. 2020 : les scénarios du futur. Paris : Des idées \& des hommes.

Resche, Catherine. 2000. «An approach to interface terminology: The example of environmental economics in English as a foreign language ». Meta: Journal des traducteurs 45/4 <http:// www.erudit.org/revue/meta/2000/v/n4/003941ar.html>. Consulté le 5/06/2013.

Rey, Alain. 1979. La terminologie : noms et notions. Paris : Presses Universitaires de France.

Rey, Alain et Juan Sager. 1995. Essays on Terminology. Amsterdam, Philadelphie : John Benjamins Publishing.

Slodzian, Monique. 1995. « Comment revisiter la doctrine terminologique aujourd'hui ? ». La Banque des mots 7, 11-18.

Slodzian, Monique. 2006. « De la mesure dans les termes ». Texto 11/1 <http://www.revuetexto.net/Inedits/Slodzian/Slodzian_Mesure.html>. Consulté le 5/06/2013.

\section{NOTES}

1. Sur cette question de l'évolution de la notion d'expertise, voir par exemple (Grandjou \& Mauz 2007) et (Millerand, Heaton, \& Proulx 2011).

2. Pour plus d'informations sur ce projet terminologique accessible en ligne: < http:// www.eila.univ-paris-diderot.fr/recherche/artes/index>.

3. Pour un «mode d'emploi » détaillé de ce mémoire: <http://www.eila.univ-paris-diderot.fr/ formations-pro/masterpro/ilts/memoire>.

4. Le texte doit comprendre au moins 20000 signes et dater de moins de trois ans.

5. Ce travail a été réalisé en 2011-2012 par Mlle Julia Hoffsteede-Prevost.

6. Travail mené en 2012-2013 par Léna Dulaurent.

7. Les contraintes de mise en page ne permettent pas de concilier lisibilité des termes et celle du schéma. Cependant, ce sont les regroupements thématiques et non les termes qui sont mis en évidence.

8. Sayhi, Sabri-Fabrice «Traduire dans le domaine de l'économie écologique: les difficultés terminologiques ». Traduire 227, décembre 2012. 


\section{RÉSUMÉS}

Le croisement des spécialités est à l'origine de l'apparition de nouveaux domaines de connaissances. La domination de la langue anglaise fait de l'anglais de spécialité un lieu d'expression privilégié de ces évolutions, obligeant l'analyste à repenser les notions de domaine, de spécialité et de terminologie. Des dictionnaires réalisés par des étudiants en master de traduction spécialisée montrent comment ces glissements affectent la conceptualisation du domaine et la manière d'enseigner la terminologie spécialisée. L'auteur étudie les conséquences, pour l'analyse terminologique, de l'émergence de domaines désignés souvent par des néologismes tels que « obsolescence programmée », à l'intersection de plusieurs problématiques, ici le management et le développement durable. Ces « hybridations » modifient l'approche des notions de terme, de définition, de collocation et la représentation graphique d'une structure terminologique. Ces glissements modifient la vision de la langue spécialisée et de son enseignement, menant d'une logique de territoire à une problématisation liée au questionnement de communautés de discours, concept à redéfinir dans ce nouveau contexte.

The constant surge of new knowledge domains tends to bring about major changes in traditional disciplinary boundaries. The domination of English makes ESP a privileged vehicle for these evolutions, forcing researchers and teachers to reconsider the concepts of domain, specialty, and terminology. Dictionaries created by specialised translation Master's students provide evidence of the way these shifts impact the way advanced students analyze terminology, and call into question conventional teaching of terminology. This study addresses the consequences of the emergence of domains which combine several fields of knowledge. One example is the field of "planned obsolescence", in which the contradictory notions of obsolescence management techniques and sustainable development are equally important. This type of hybridation has an impact on such basic concepts as "term", "definition", "collocation", and the graphic representation of a terminological structure. These shifts modify the vision of specialised language and the way it is taught, and move the focus from territories to communities The very idea of discourse communities needs reexamining in this light.

\section{INDEX}

Keywords : corpus analysis, domain, language for specific purposes, specialised translation, specialised field, terminology

Mots-clés : analyse de corpus, domaine, langue de spécialité, terminologie, traduction spécialisée

\section{AUTEUR}

\section{GENEVIÈVE BORDET}

Geneviève Bordet est maître de conférences à l'Université Paris Diderot et membre du laboratoire CLILLAC-ARP (EA 3967). Sa recherche porte sur la rédaction du discours scientifique en anglais par des locuteurs natifs et non natifs. Elle a publié dans diverses revues, comme Discours (2011). gbordet@eila.univ-paris-diderot.fr 